$1-1-2016$

\title{
Modeling Cross-Contamination During Poultry Processing: Dynamics in The Chiller Tank
}

\author{
Daniel Munther \\ Cleveland State University, d.munther@csuohio.edu \\ Xiaodan Sun \\ Xi'an Jiaotong University \\ Yanni Xiao \\ Xi'an Jiaotong University \\ Sanyi Tang \\ Shaanxi Normal University \\ Helio Shimozako \\ York University
}

See next page for additional authors

Follow this and additional works at: https://engagedscholarship.csuohio.edu/scimath_facpub

Part of the Mathematics Commons

How does access to this work benefit you? Let us know!

\section{Repository Citation}

Munther, Daniel; Sun, Xiaodan; Xiao, Yanni; Tang, Sanyi; Shimozako, Helio; Wu, Jianhong; Smith, Ben A.; and Fazil, Aamir, "Modeling Cross-Contamination During Poultry Processing: Dynamics in The Chiller Tank" (2016). Mathematics Faculty Publications. 206.

https://engagedscholarship.csuohio.edu/scimath_facpub/206

This Article is brought to you for free and open access by the Mathematics and Statistics Department at EngagedScholarship@CSU. It has been accepted for inclusion in Mathematics Faculty Publications by an authorized administrator of EngagedScholarship@CSU. For more information, please contact library.es@csuohio.edu. 


\section{Authors}

Daniel Munther, Xiaodan Sun, Yanni Xiao, Sanyi Tang, Helio Shimozako, Jianhong Wu, Ben A. Smith, and Aamir Fazil 


\title{
Modeling cross-contamination during poultry processing: Dynamics in the chiller tank
}

\author{
Daniel Munther, Xiaodan Sun, Yanni Xiao, Sanyi Tang, Helio Shimozako, \\ Jianhong Wu, Ben A. Smith, Aamir Fazil
}

\section{Introduction}

Poultry contamination by bacterial pathogens such as Salmo nella, Campylobacter and Escherichia coli O157:H7, continues to pose a serious threat to public health both in Canada and on the global scale. According to the World Health Organization, 25\% of food borne outbreaks are closely associated with cross contamination events involving deficient hygiene practices, contaminated equip ment, contamination via food handlers, processing, or inadequate storage (Carrasco, Morales Rueda, \& Garcia Gimeno et al., 2012). As processing has been highlighted as a pivotal juncture in the supply chain, both for preventing and potentially promoting cross contamination, researchers have conducted numerous studies, attempting to determine pathogen prevalence and concentration at various processing stages. However, the underlying mechanisms of cross contamination are still poorly understood and, furthermore, many studies evaluating the efficacy of intervention strategies during processing have presented inconsistent and even contradictory results. One reason for such issues is that studies were conducted at the lab or pilot scale under specific conditions that leave their results difficult to synthesize (Bucher et al., 2012).

In this work, part one of a series of studies, we develop a mathematical model to gain insight into the main mechanisms of chlorine decay and cross contamination during the chilling pro cess. This approach is important because of its ability to test mechanistic hypotheses as well as to help streamline experiments that would other wise be expensive both financially and tempo rally. More specifically, modeling informed insights can be used as cost effective tools to help describe the mechanisms driving cross contamination, and to establish unambiguous, quantifiable links between processing control parameters (such as chiller water temperature, wash time, chlorine concentration, carcass to water volume ratio, etc.) and pathogen prevalence and concentration. In turn, the quantified connections between control parameters and pathogen dynamics can provide invaluable information in terms of testing control strategies to keep pathogen levels below thresholds.

While our focus is the chiller process of a typical modernized Canadian poultry inspection program plant (high speed), our model can be easily generalized to chiller processes in other locales. Also, 
the modeling framework and techniques can be modified to describe similar mechanisms in the process of defeathering, evis ceration and scalding. We describe the background and modeling formulation in Section 2. In Section 3 we apply our model to generic, non pathogenic E. coli contamination of broiler carcasses, discuss detailed parameter estimation, and perform sensitivity analysis. Using the results of the sensitivity analysis, we discuss thresholds within which cross contamination and chlorine control play a lesser role as well as when cross contamination may pose a more significant risk. Also, in Section 3, we compare model pre dictions for $E$. coli levels on poultry exiting the chiller tank when free chlorine (FC) input is used at $50 \mathrm{mg} / \mathrm{l}$ or not at all. These results are given in terms of USDA baseline values. In addition, we examine the dynamics of FC inactivation via the organic load in chiller red water, i.e., chiller water that has been exposed to poultry carcasses, organic material and possibly pathogens. In the final section, we suggest some quantified rules of thumb for managing cross contamination issues and discuss the feasibility of developing more complex models and of simplifying the complexity of cross contamination models for relatively easy implementation.

\section{Background and chiller model}

Canada has a variety of poultry processing operations, ranging from smaller traditional type processing to state of the art, high speed operations. In this work, we consider a typical modernized poultry processing plant (high speed), which covers most of the Canadian slaughter production (based on personal communication with CFIA officers, which we will reference from now on as [P]). Essentially, our processing framework involves a poultry slaughter establishment which operates under the CFIA approved Modern ized Poultry Inspection Program (MPIP); see CFIA (2014) for more information. This perspective leads to several assumptions that guide our model formulation. These include (1) the typical weight of a carcass is $2 \mathrm{~kg}$; (2) the typical processing speed is 180 carcasses/ min; (3) the average dwell time of carcasses in the chiller tank is $45 \mathrm{~min}$; (4) red water is not recycled, rather the set up involves fresh water intake at the beginning of the chiller tank, with over flow at the end; (5) a maximum of $50 \mathrm{ppm}(\mathrm{mg} / \mathrm{l})$ of free chlorine (FC) is added (if any) at the beginning of the chiller tank, and mixed with incoming fresh water; and (6) due to model simplification and a lack of data, we assume that organic matter and microbes do not bind/attach to the tank surfaces.

Our model is built around two main types of mechanisms: (i) those that involve typical processing procedures for immersion chilling in high speed poultry processing facilities in Canada and (ii) bacteria transfer, bacteria inactivation, and water chemistry dy namics during the chilling process. Refer to Table 2 for a list of parameters corresponding to type (i) and (ii). To be clear, the pa rameters involved with the particular processing assumptions and dynamics, as in (i), are what specifies our model for Canadian poultry programs. The mechanisms under type (ii) are general mechanisms that are expected in a typical large scale immersion chilling procedure that is utilized during poultry processing in many locales, not just Canada. Therefore, in this section as well as Section 3, where we apply our model to generic E. coli contami nation, data used to quantify the type (ii) mechanisms need not necessarily be Canadian.

We now formulate the chiller model in several steps.

\subsection{The carcass dynamics and total suspended solids}

We assume that the incoming rate of chicken carcasses to the tank is $N(\mathrm{~kg} / \mathrm{min})$ and the chickens spend on average $1 / d_{p}(\mathrm{~min})$ in the tank. These two assumptions lead to the following equation for $P$, the total $\mathrm{kg}$ of chicken carcasses in the tank at time $t \geq 0$ (min):

$P^{\prime} \quad N \quad \varepsilon d_{p} P$,

where

$\varepsilon \begin{cases}0, & t \leq \frac{1}{d_{p}} \\ 1, & t>\frac{1}{d_{p}} .\end{cases}$

Note that ' is the derivative with respect to time and the function $\varepsilon$ ensures that no carcasses will leave the tank before the "average" wash time $1 / d_{p}$ has elapsed.

As the chickens enter and move through the chiller tank, they release high amounts of organic material (in the form of blood, fat, protein, etc.) into the water. Such material is important because it alters chiller water chemistry as well as microbial counts (Russell, 2012). We represent the organic material in the chiller tank at time $t>0$ by $J(\mathrm{~kg})$. In order to relate this to the total suspended solids (concentration), we consider $J / T_{V}$, where $T_{V}$ is the total tank volume in $\mathrm{ml}$. For simplicity, we assume that the amount of organic material coming in to the water is proportional to the incoming rate of chicken carcasses $N(\mathrm{~kg} / \mathrm{min})$ and this is represented by $q \in(0,1)$. Note that in reality, the amount of organic material shed from in dividual carcasses may be independent of one another. Also, we assume, via the flow through the tank, that the organic material spends on average $1 / d_{p}$ minutes in the tank. Therefore we build the following equation for $J$ :

$J^{\prime} \quad q N \quad \varepsilon d_{p} J$.

\subsection{Average microbial load on carcasses and organic material in the tank}

One of the key purposes of the model is to understand the dy namics of the average microbial load on both the poultry and the organic material in the chiller tank. To do so, we represent the average microbial load $(\mathrm{CFU} /(\mathrm{kg} \mathrm{ml}))$ on the chicken and organic material in the tank at time $t>0$, by $v_{p}$ and $v_{j}$, respectively. Notice that the units for $v_{p}$ and $v_{j}$ are $(\mathrm{CFU} /(\mathrm{kg} \mathrm{ml}))$ since we scale the average bacteria load per $\mathrm{kg}$ by the tank volume $T_{V}$. For modeling purposes, it is convenient to scale by the tank volume and this scaling should not be connected with bacterial concentration measurements taken from typical rinse procedures used to quan tify the microbial load on a pre or post chill carcass. For instance, the USDA conducted studies using a $400 \mathrm{ml}$ carcass rinse in order to determine $E$. coli levels on individual poultry carcasses during processing and reported their results in units CFU/ml (USDA, 2012).

We assume that the chickens enter the chiller process with an average level of $\sigma \mathrm{CFU} / \mathrm{kg}$. Upon entering the tank, a certain fraction of this contamination level initially sheds into the chiller water. Let this fraction be $\rho$ and so $0<\rho<1$. Also, as the carcasses move through the chiller tank, we suppose that continued microbial shedding occurs at a rate $b v_{p}$, where $b(1 / \mathrm{min})$ is the shedding parameter (i.e., the shedding rate is proportional to the current average contamination level on the poultry). In addition, bacterial attachment occurs via contact between a carcass and microbials in the chiller water. If we let $W(\mathrm{CFU} / \mathrm{ml})$ be the microbial concen tration in the chiller water at time $t$, then we assume this attach ment occurs at a rate $\beta W$, where $\beta(1 /(\mathrm{kg} \mathrm{min}))$ is the binding parameter. 
In addition to shedding/binding, we consider the inactivation of microbes on carcass surfaces via free chlorine (FC) contact during the chiller process. While the effective contact of FC with carcass surfaces (and therefore with microbes attached to carcass surfaces) during immersion chilling is, to our knowledge, not well docu mented in the literature, there are multiple studies quantifying inactivation rates of microbes in solution via FC; see (Helbling \& VanBriesen, 2007; Zhang, Luo, Zhou, Wang, \& Millner, 2015) and references therein. If we let $k_{w}>0$ be the inactivation rate of mi crobes in the chiller water, then we argue that the inactivation rate via FC of microbes on carcass surfaces can be written as $\alpha k_{w}$, where $\alpha \in(0,1)$. For instance, in the fresh produce industry, studies have concluded that surface characteristics can reduce effective contact of chemical sanitizers during wash cycle protocols (Adams, Hartley, \& Cox, 1989; Gil, Selma, López Gálvez, \& Allende, 2009). Since carcass surfaces are irregular and this is an important factor in determining contamination levels (Thomas \& McMeekin, 1980), similar to the results from fresh produce studies, FC contact with microbes attached to carcass surfaces should be significantly less than FC contact with microbes in the chiller water. Combining these ideas, the average decrease of the microbial load on carcasses is given by $\alpha k_{w} v_{p} C$, where we assume that this decrease is propor tional to the product of the current microbial load and the FC concentration $C(\mathrm{mg} / \mathrm{L})$.

Finally, taking into account the fact that $1 / d_{p}$ is the average wash time (and assuming that the natural death rate of the microbes attached to the poultry and organic material is zero (Russell, 2012)) our equation for $v_{p}$ becomes:

$v_{p}^{\prime} \quad \frac{(1 \quad \rho) \sigma N}{P T_{V}}+\beta W \quad b v_{p} \quad d_{p} v_{p} \quad \alpha k_{w} v_{p} C$.

In a similar manner, we can construct an equation for $v_{j}$ as follows:

$v_{j}^{\prime} \quad \frac{(1 \quad \rho) q \sigma N}{J T_{V}}+\beta W \quad b v_{j} \quad d_{p} v_{j} \quad \alpha k_{w} v_{j} C$.

Notice that the carcass surface temperatures during the initial cooling phase of chilling may promote microbial growth on carcass surfaces. Please refer to Section 4 for reasons as to why we do not include this dynamic in Equations (3) and (4) for specific applica tion to E. coli contamination.

\subsection{Dynamics in the chiller water}

The two main variables we examine include $W$, the microbial concentration in the chiller water, and $C(\mathrm{mg} / \mathrm{L})$, the FC concen tration in the water. Assuming that red water is not filtered or recycled, bacteria do not multiply in the water because of the low temperature, $\leq 4{ }^{\circ} \mathrm{C}$ as per Canadian Food Inspection Agency (CFIA) regulations, and bacterial survival in the water is expected (Ratkowsky, Olley, McMeekin, \& Ball, 1982; Wang \& Doyle, 1998; CFIA, 2014), $W$ depends on four things: (i) microbes shed into the water (due to sheer forces in the water, etc.), (ii) microbes in water attaching to poultry or organic material, (iii) microbes inactivated by FC and (iv) the flow rate of water in/out of the tank. While there may be concentration differences along the length of the tank, for simplicity we assume complete mixing for the dynamics in (i) (iv). Observe that injured bacteria cells might also be represented in this model, given the assumption that they shed from/adhere to car casses at the same rates as other intact/viable cells. However, from a modeling standpoint we do not differentiate between injured and viable cells.

In terms of quantifying the above mechanisms, for instance, the inactivation rate for microbes in water due to FC is given by
$W^{\prime} \quad k_{w} C W$, where $k_{w}(\mathrm{l} /(\mathrm{mg} \min ))$ is the inactivation rate parameter. Notice, the change in $W$ at time $t$ is proportional to $C W$, illustrating the common mass action assumption for such chemical reactions (Deborde \& von Gunten, 2008). Putting together this idea with (i) (iv), the equation for $W$ is:

$$
W^{\prime} \quad(1+q) \frac{\sigma \rho N}{T_{V}}+b v_{j} J+b v_{p} P \quad \beta P W \quad \beta J W \quad k_{W} C W \quad g \frac{W}{10^{-3} T_{V}}
$$

where the first term $(1+q) \sigma \rho N / T_{V}$ reflects the amount of contamination that initially sheds from the poultry into the water and the last term $g W / 10^{-3} T_{V}$, reflects the concentration of microbes that exit the tank with the outflow. Notice, we assume the tank volume $T_{V}$ is constant in time, so inflow outflow. Assuming that $l$ liters of fresh water are added to the tank per carcass and each carcass on average weighs $m \mathrm{~kg}, \mathrm{~g} \quad \mathrm{~N}(\mathrm{~kg} / \mathrm{min})^{*}(1$ carcass $/ \mathrm{m} \mathrm{kg})$ * $l(1$ freshwater/carcass $) \quad \mathrm{Nl} / \mathrm{m}(\mathrm{l} / \mathrm{min})$ is the addition rate of fresh water per carcass.

In terms of the equation for $C$, we have:

$$
C^{\prime} \quad c_{1} \frac{g}{10^{-3} T_{V}} \quad d_{C} C \quad k_{c} W C \quad \alpha k_{c} v_{j} J C \quad \alpha k_{c} v_{p} P C \quad h_{J} J C \quad \frac{C}{10^{-3} T_{V}},
$$

where $c_{1}(\mathrm{mg} / \mathrm{l})$ is the FC concentration of the input water, $g$ is as above, and so $c_{1} g / 10^{-3} T_{V}(\mathrm{mg} /(1 \mathrm{~min}))$ measures the rate of increase of FC in the water. The natural decay rate of FC in the tank is rep resented by $d_{c}(1 / \mathrm{min})$. Also, $k_{c}(\mathrm{ml} /(\mathrm{CFU} \min ))$ reflects the rate at which chlorine is oxidized or degraded due to inactivating mi crobes in the water, $\alpha k_{c}$ is the rate at which FC is degraded due to inactivating microbes on carcass surfaces, and $h_{J}(1 /(\mathrm{kg}$ min $))$ is the rate at which the organic material in the tank decreases the FC through chemical binding. For the terms involving $k_{c}$ and $h_{J}$ we assume that the decrease in FC concentration at time $t$ is propor tional to the product of the respective interacting "species", and therefore $k_{c}, \alpha k_{c}$ and $h_{J}$ are types of second order rate constants. Finally, $g C / 10^{-3} T_{V}$ illustrates the loss of FC due to outflow of water from the tank.

\subsection{Complete model}

Putting together the six equations above, our model becomes:

$$
\begin{aligned}
& J^{\prime} \quad q N \quad \varepsilon d_{p} J, \\
& v_{j}^{\prime} \quad \frac{(1 \quad \rho) q \sigma N}{J T_{V}}+\beta W \quad b v_{j} \quad d_{p} v_{j} \quad \alpha k_{w} v_{j} C, \\
& P^{\prime} \quad N \quad \varepsilon d_{p} P, \\
& v_{p}^{\prime} \quad \frac{(1 \quad \rho) \sigma N}{P T_{V}}+\beta W \quad b v_{p} \quad d_{p} v_{p} \quad \alpha k_{w} v_{p} C \\
& W^{\prime} \quad(1+q) \frac{\sigma \rho N}{T_{V}}+b v_{j} J+b v_{p} P \quad \beta P W \quad \beta J W \quad k_{w} C W \quad g \frac{W}{10^{-3} T_{V}}, \\
& C^{\prime} \quad c_{1} \frac{g}{10^{-3} T_{V}} \quad d_{C} C \quad k_{C} W C \quad \alpha k_{C} v_{j} J C \quad \alpha k_{c} v_{p} P \quad h_{J} J C \quad g_{\frac{C}{10^{-3} T_{V}}} .
\end{aligned}
$$

See Table 1 for a concise description of the model (7) variables.

\subsection{Model properties}

Note that system (7) is positively invariant for all non negative, not identically zero initial conditions. This essentially means that for each set of non negative, not identically zero initial conditions, 
Table 1

Model variables.

\begin{tabular}{ll}
\hline Variable & Description \& units \\
\hline$J$ & Amount of organic material in tank at time $t(\mathrm{~kg})$ \\
$v_{j}$ & Average microbial load on organic material in tank at time \\
& $t(\mathrm{CFU} /(\mathrm{kg} \mathrm{ml}))$ \\
$P$ & Amount of poultry in tank at time $t(\mathrm{~kg})$ \\
$v_{p}$ & Average microbial load on poultry in tank time $t(\mathrm{CFU} /(\mathrm{kg} \mathrm{ml}))$ \\
$W$ & Microbial concentration in chiller water at time $t(\mathrm{CFU} / \mathrm{ml})$ \\
$C$ & FC concentration in chiller water at time $t(\mathrm{mg} / \mathrm{l})$ \\
\hline
\end{tabular}

there exists a unique solution to (7) which stays positive in each component for all positive time (i.e. we can meaningfully ascribe physical units to each component of the solution). Furthermore, such solutions to (7) are not unbounded in finite time. Combining these ideas, we see that system (7) can unambiguously describe the variables we have associated to a continuous poultry chilling pro cess with potential cross contamination dynamics.

In addition, our system has a positive equilibrium state which we denote by

$\Sigma \quad\left(J^{*}, v_{j}^{*}, P^{*}, v_{p}^{*}, W^{*}, C^{*}\right)$.

This equilibrium state $\Sigma$ is independent of time and according to numerical calculations (not shown) it attracts all solutions with non negative, not identically zero initial conditions. This means that as time increases, biologically relevant solutions (as described above) move closer in value to $\Sigma$. The equations for each coordinate of $\Sigma$, in terms of model parameters, are given below (note that some are implicitly given for the sake of clarity).

$$
\begin{aligned}
& J^{*} \quad \frac{q N}{d_{p}} \\
& P^{*} \quad \frac{N}{d_{p}} \\
& v_{p}^{*} \quad v_{j}^{*} \quad \frac{(1 \rho) d_{p} \sigma}{T_{V}\left(b+d_{p}+\alpha k_{w} C^{*}\right)}+\frac{\beta W^{*}}{b+d_{p}+\alpha k_{W} C^{*}} \\
& W^{*} \frac{\frac{(1+q) \sigma \rho N}{T_{V}}+b\left(v_{j}^{*} J^{*}+v_{p}^{*} P^{*}\right)}{\beta\left(P^{*}+J^{*}\right)+k_{w} C^{*}+\frac{g}{10^{3} T_{V}}} \\
& C^{*} \frac{c_{1} g}{\left(10^{-3} T_{V}\right)\left(d_{c}+k_{c} W^{*}+\alpha k_{c} v_{j}^{*} J^{*}+\alpha k_{c} v_{p}^{*} P^{*}+h_{J} J^{*}+\frac{g}{10^{3} T_{V}}\right)}
\end{aligned}
$$

\section{Application of model to $E$. coli cross-contamination during immersion chilling}

Due to the fact that the we have a relatively complete data set for generic E. coli, both bacteria levels and transfer during industrial chiller processes (Cavani, Schocken Iturrino, Garcia, \& de Oliveira, 2010; Northcutt, Smith, Huezo, \& Ingram, 2008; Tsai, Schade, \& Molyneux, 1992) and chlorine inactivation (Helbling \& VanBriesen, 2007), we tailor our model to address the specific dynamics asso ciated to the chiller water chemistry and cross contamination of broiler carcasses contaminated with non pathogenic E. coli. For the parameter ranges specific to this situation, the solutions of system
(7) approach $\Sigma$ on the order of 200-250 min, which means during a typical $8 \mathrm{~h}$ shift, if there is little variation in the average $E$. coli load on carcasses, i.e. $\sigma \approx$ constant, the model predicts that contamina tion levels in the water and on the poultry in the tank will equili brate. Practically, this gives us mathematical justification to simplify the dynamics in the tank and consider only the equilib rium solution $\Sigma$ of (7). However, since the parameters involved with E. coli contamination are not precisely known, we want to under stand how sensitive $\Sigma$ is to the model parameters. This sensitivity analysis is vital for making informed conclusions for $E$. coli control as we illustrate in the following sections.

Note that $\sigma$ may vary significantly during an $8 \mathrm{~h}$ shift and therefore depending on certain time intervals, contamination levels in the chiller water and on the poultry may vary instead of equili brating. Realistically, then, $\sigma$ should depend on time. However, in order to build control strategies and rules of thumb for treating such cases, we first seek results that act as a reference point. That is, we first determine a range for $\sigma$ in which cross contamination plays a significant role and gain an understanding of which parameters play dominant roles in contributing to cross contamination at equilibrium. We do this via sensitivity analysis, assuming $\sigma$ is constant but randomly selected from within its range. Please see Section 3.1 for details concerning this analysis as well as Section 3.2.2 and Section 4 for situations where $\sigma$ may vary as a function of time and how the model (7) can be applied to quantitative micro bial risk assessment (QMRA).

\subsection{Parameter baseline and range estimation for $E$. coli contamination}

\subsubsection{Parameters specific to Canadian processing}

Referring to Table 2, specific processing parameter values were obtained from personal communication with CFIA officers, as referenced by [P]. Refer to the beginning of Section 2 for the details of Canadian high speed chilling specifications. Note that the other studies referenced for these same parameters in Table 2 confirm the uniformity of some of these assumptions for immersion chilling in other locations such as the U.S. and Brazil.

\subsubsection{Average E. coli load on incoming carcasses}

$\sigma$ : Following (Northcutt et al., 2008), we can set a baseline value for $\sigma$, the average microbial load on incoming carcasses (CFU $/ \mathrm{kg}$ ). From (Northcutt et al., 2008), the rinsing procedure to quantify the bacterial load on poultry prior to the chilling process, indicates that the average $E$. coli level for incoming carcasses is $10^{2.6} \mathrm{CFU} / \mathrm{ml}$ in the rinsate. Given a $100 \mathrm{ml}$ rinse, this translates to roughly $10^{4.6} \mathrm{CFU}$ on the average carcass. Assuming the average carcass weight is $2 \mathrm{~kg}$, $\sigma \quad 10^{4.6} / 2 \approx 2 \times 10^{4} \mathrm{CFU} / \mathrm{kg}$. For sensitivity analysis, we establish the following range for $\sigma, 10^{3}$ to $10^{6} \mathrm{CFU} / \mathrm{kg}$ based on $E$. coli data in Cavani et al. (2010).

\subsubsection{Shed rate of E. coli from carcasses to chiller water}

$b$ : From Northcutt et al. (2008), we can estimate $b$ by comparing the pre chill bacteria load on a carcass and the post chill bacteria load. Following the rinse procedure in (Northcutt et al., 2008), the pre chill E. coli load recovered from an average single carcass was $10^{2.6} \mathrm{CFU} / \mathrm{ml}$ and the post chill load was $10^{1.1} \mathrm{CFU} / \mathrm{ml}$. By conser vation of the E. coli population, and considering a $45 \mathrm{~min}$ average wash time, we estimate the shed rate to be:

b $\quad \frac{\ln \left(10^{1.1} / 10^{2.6}\right)}{45} \quad .077 \quad 1 / \mathrm{min}$.

In terms of a range for $b$, we use 0.04 to 0.1 . Considering that a 
Table 2

Baseline parameters values for application to E. coli. Parameters with reference [P], correspond to information obtained from personal communication with CFIA officers. Currently there is no documented data for these references marked with [P]. Parameters $l$ and $g$ are extrapolated from guidelines in (CFIA, 2014).

\begin{tabular}{|c|c|c|c|}
\hline Type & Parameter/Reference & Description & Values/Units \\
\hline \multirow[t]{7}{*}{ (i) } & $\begin{array}{l}T_{V} \\
\text { (Cavani et al., 2010) }\end{array}$ & Tank volume & $5 \times 10^{7} \mathrm{ml}$ \\
\hline & $\begin{array}{l}\text { N } \\
\text { (Cavani et al., 2010) } \\
\text { (Northcutt et al., 2008) }\end{array}$ & Carcass process rate & $360 \mathrm{~kg} / \mathrm{min}$ \\
\hline & $\begin{array}{l}1 / d_{p} \\
{[P],(\text { Northcutt et al., 2008) }}\end{array}$ & Average wash time & $45 \mathrm{~min}$ \\
\hline & $\begin{array}{l}l \\
(\text { CFIA, 2014) }\end{array}$ & Fresh water/carcass & $1.7 \mathrm{l} /$ carcass \\
\hline & $\begin{array}{l}m \\
{[\mathrm{P}]}\end{array}$ & Average carcass weight & $2 \mathrm{~kg}$ \\
\hline & $\begin{array}{l}g \\
(\text { CFIA, 2014) }\end{array}$ & input water rate & $306 \mathrm{l} / \mathrm{min}$ \\
\hline & $\begin{array}{l}c_{1} \\
\text { (USDA, 2012; CFIA, 2014) }\end{array}$ & input FC concentration & $050 \mathrm{mg} / \mathrm{l}$ \\
\hline \multirow[t]{10}{*}{ (ii) } & $\begin{array}{l}d_{c} \\
\text { (Li, Gu, Qi, Ukita, \& Zhao, 2003) }\end{array}$ & FC decay rate & $4.1 \times 10^{5} \min 1$ \\
\hline & $\begin{array}{l}\sigma \\
\text { (Northcutt et al., 2008) }\end{array}$ & Prechill carcass load & $2 \times 10^{4} \mathrm{CFU} / \mathrm{kg}$ \\
\hline & $\begin{array}{l}\rho \\
\text { estimated }\end{array}$ & Initial shed fraction & 0.15 \\
\hline & $\begin{array}{l}q \\
\text { (Northcutt et al., 2008) } \\
\text { (Tsai et al., 1992) }\end{array}$ & Organic material fraction & 0.011 \\
\hline & $\begin{array}{l}\beta \\
\text { (Munther \& Wu, 2013) } \\
\text { (Northcutt et al., 2008) }\end{array}$ & Microbial attachment rate & $0.01(\mathrm{~kg} \mathrm{~min})^{1}$ \\
\hline & $\begin{array}{l}b \\
\text { (Northcutt et al., 2008) }\end{array}$ & Microbial shed rate & $0.077 \min 1$ \\
\hline & $\begin{array}{l}\alpha \\
\text { estimated }\end{array}$ & Fraction for FC kill rate on carcass & $0.001 \quad 0.1$ \\
\hline & $\begin{array}{l}k_{w} \\
\text { (Helbling \& VanBriesen, 2007) }\end{array}$ & FC kill rate in water & $216 \mathrm{l} /(\mathrm{mg} \min )$ \\
\hline & $\begin{array}{l}k_{c} \\
\text { (Helbling \& VanBriesen, 2007) }\end{array}$ & FC decay rate via killing & $0.0069 \mathrm{ml} /(\mathrm{CFU} \mathrm{min})$ \\
\hline & $\begin{array}{l}h_{J} \\
\text { (Tsai et al., 1992) }\end{array}$ & FC oxidation rate & $0.0017(\mathrm{~kg} \mathrm{~min})^{1}$ \\
\hline
\end{tabular}

carcass undergoes an average chilling time of $45 \mathrm{~min}$, this corre sponds to a 1 to $2 \log$ reduction on the poultry.

\subsubsection{FC inactivation kinetics}

$k_{c}$ and $k_{w}$ : From Helbling and VanBriesen (2007), we have that the " $3 \log _{10}$ " $\mathrm{CFU} / \mathrm{ml}$ inactivation contact time is given by $.032 \pm .009(\mathrm{mg} / \mathrm{l}) \mathrm{min}$. That is, it takes $0.032 \mathrm{mg} / \mathrm{l}$ of FC concen tration to inactivate $10^{3} \mathrm{CFU} / \mathrm{ml}$ of $E$. coli in solution in one minute. The study in Helbling and VanBriesen (2007) indicates that E. coli is very reactive with FC and the "contact time" is calculated by inte grating the FC concentration curve over the time interval $\left[t_{0}, t_{3}\right]$, i.e., the time interval it takes to reduce the microbial concentration by $10^{3}$.

Considering the units of $k_{w}$ and $k_{c}$, and the fact that it takes $0.032 \mathrm{mg} / \mathrm{l}$ of FC to eliminate $10^{3} \mathrm{CFU} / \mathrm{ml}$ of $E$. coli in one minute, we calculate:

\section{$k_{w} \quad 3.125 \times 10^{4} k_{c}$.}

Also, as bacteria are organic substances, we can model their inactivation by FC using a second order rate reaction (Deborde \& von Gunten, 2008), $W \quad k_{w} C W$. Considering this equation on the time interval $\left[t_{0}, t_{3}\right]$, we can solve for $k_{w}$ as follows:

$k_{w} \frac{\ln \left(W\left(t_{0}\right) / W\left(t_{3}\right)\right)}{\int_{t_{0}}^{t_{3}} C(s) d s} \quad \frac{\ln \left(10^{3}\right)}{.032} \quad 215.867$.
Using the relationship above, we see that $k_{c} \quad 0.0069$. From the range given for the contact time above, we find that $k_{w} \in[150,300]$, while $k_{c}$ ranges from about 0.0048 to 0.0096 . Performing similar calculations with inactivation of $E$. coli 0157:H7 data from (Zhang et al., 2015), we find that $k_{w} \approx 276$ and $k_{c} \approx .02$. Here we use the result that $5 \log _{10}$ reduction is achieved in $0.25 \mathrm{~s}$ with $10 \mathrm{mg} / \mathrm{l}$ of FC (Zhang et al., 2015). Because $k_{c}$ barely affects model outputs as it varies across its range, for simplicity we fix $k_{c} \quad .0069$ and do not include it in the sensitivity analysis.

In terms of inactivation of $E$. coli on carcass surfaces, we assume that the rate is $\alpha k_{w}$ and the loss of FC due to this inactivation, since $k_{c}$ is proportional to $k_{w}$, is $\alpha k_{c}$. While $\alpha$ is not precisely known, considering the discussion in Section 2.2, we assume that it is at most 0.1 . For the sensitivity analysis, we assume that $\alpha$ ranges from 0.001 to 0.1 .

\subsubsection{Organic material in the chiller water}

$q$ : From Tsai et al. (1992), the total suspended solids in the chiller water is $0.35 \%$ (i.e. about $3500 \mathrm{mg} / \mathrm{l}$ ) (this value is similar to the initial measuring station in the tank (Northcutt et al., 2008)). With our tank volume given by $T_{V} 5 \times 10^{7} \mathrm{ml}$, this translates to about $175 \mathrm{~kg}$. Using the total suspended solids to estimate the organic material in the tank, $J(t)$ should equilibrate to about $175 \mathrm{~kg}$. We know that the positive equilibrium value of $J \quad q N / d_{p}$ without filtering. This implies that $q \approx 175 /\left(45^{*} 360\right) \quad 0.011$.

Since $N$ and $d_{p}$ are fixed from our processing assumptions, we allow $q$ to vary from 0.005 to 0.03 as this means that $J$ varies from about 80 to $490 \mathrm{~kg}$. 


\subsubsection{FC oxidation rate via organic material in tank}

$h_{j}$ : We want to estimate the rate of chlorine reaction with the organic material in the chiller water. From our model, we use the following equation:

$C^{\prime} \quad h_{J} J C$.

From Tsai et al. (1992), the chiller water is assumed to have the total suspended solids at equilibrium, $J 3500 \mathrm{mg} / \mathrm{l}$ (or $0.35 \%$ ). Assuming, as above, $T_{V} \quad 5 \times 10^{7} \mathrm{ml}$, we have that for large enough $t, J(t) \equiv J \quad J \frac{T_{V} \mathrm{l}}{10^{6} \mathrm{mg}} 175 \mathrm{~kg}$. Substituting this into the model, we solve to get

$$
C(t) \quad C_{0} e^{-h_{J} J t}
$$

Referring to the data in Table 5 of Tsai et al. (1992), we see that chlorine depletion from organic material has both a "fast" and "slow" kinetic. For our purposes, we consider only the fast kinetic as we have a continuous flow of chlorine and organic material entering the chill tank. From Tsai et al. (1992), the average of this fast kinetic is $0.29 / \mathrm{min}$ with standard deviation 0.10 . Combining this with the rate in (13), leads to $h_{J} J \quad 0.29 \pm .10$. Since $J \quad 175 \mathrm{~kg}$, our baseline value for $h_{J} \quad .0017$ and the range is $h_{J} \in$ [0.0011,0.0022]. Note, the residual chlorine data from Tsai et al. (1992) is not the same as FC, however, we assume that the resid ual chlorine is proportional to the FC and therefore the decay rate for both types will be given by $h_{J}$.

\subsubsection{Binding rate of E. coli to poultry in tank}

$\beta$ : To estimate the binding rate of $E$. coli in suspension in the process water to the poultry during chilling, we adopt the "trans mission rate" perspective that is common to disease models. In a disease model with a well mixed population, this rate is based on the number of successful contacts an infective individual makes with the susceptible population (Brauer, 2008). For the chilling process, the number of contacts depends on (a) the poultry to water ratio, (b) the average dwell time of the poultry in the tank and (c) the "path" the carcasses take through the tank. We suppose that the tank is $9 \mathrm{~m}$ long (Northcutt et al., 2008) and its volume is $T_{V} \quad 5 \times 10^{7} \mathrm{ml}$. Because the equilibrium amount of poultry $P^{*} \quad \mathrm{~N} /$ $d_{p} \quad 16200 \mathrm{~kg}$, the poultry to water ratio is $P^{*} / T_{V} \quad 0.324 \mathrm{~kg} / \mathrm{l}$.

We want to know how many liter "cubes" of contaminated water this $0.324 \mathrm{~kg}$ of poultry "hits" as it travels through the tank. Assuming the $0.324 \mathrm{~kg}$ poultry unit travels straight through the tank, and because a liter cube of water has a side dimension of $0.1 \mathrm{~m}$, the poultry unit "hits" about 90 cubes of water. Therefore, $1 \mathrm{~kg}$ of poultry "hits" about $270 \mathrm{~L}$ cubes during its $45 \mathrm{~min}$ trip through the tank. We describe $\beta$ as follows:

$$
\beta \quad \frac{\frac{270 \mathrm{hits}}{45 \mathrm{~min}}}{\mathrm{~kg}} \mu,
$$

where $\mu$ is the probability of successful E. coli attachment. Currently we have no data for $\mu$, but we estimate it to be between $.02 \%$ and $2 \%$ success. See Munther \& Wu (2013) for a discussion on the proba bility of $E$. coli attachment to lettuce during a commercial produce wash. In that context, $\mu \approx 1 \%$. Putting these ideas together indicates that $\beta \in[0.001,0.1]$.

\subsection{Results from sensitivity analysis}

In order to understand how parameter variations affect $W^{*}, v_{p}^{*}$ and $C^{*}$ (i.e. the equilibrium $E$. coli levels in the chiller water, on the poultry and the equilibrium FC level), we use Latin hypercube sampling to build a matrix of parameter input values (see Tables 2 and 3). These input values are then fed into our model (7) and linked with the corresponding outputs for $W^{*}, v_{p}^{*}$ and $C^{*}$. Using a sample size of $n \quad 1000$, we calculate the partial rank correlation coefficients (PRCCs) corresponding to each parameter. Briefly, the PRCC values quantify the degree of monotonicity between respec tive parameters and outputs. For more details concerning this analysis, please refer to (Marino, Hogue, Ray, Krischner, \& Zhao, 2008). Observe that for a complete uncertainty and sensitivity analysis, we should also perform an extended Fourier amplitude sensitivity test (eFAST), however, more relevant data for certain parameter ranges is needed in order to justify an extensive sensi tivity analysis.

Fig. 1 illustrates the PRCC values using the baseline and range values for corresponding parameters coming from Tables 2 and 3. From Fig. 1(A) and (C), we first notice that $W^{*}$ and $v_{p}^{*}$ are strongly influenced by $\sigma(\mathrm{CFU} / \mathrm{kg})$, the average $E$. coli load on pre chilled poultry. This is a logical result, as increasing the pre chiller mi crobial load on the poultry will in general lead to an increase in microbial concentration in the chiller water as well as on carcasses during chilling. We quantify the average $E$. coli load (during chill ing) on the poultry, $v_{p}^{*}(\mathrm{CFU} /(\mathrm{kg} \mathrm{ml}))$, as the time independent expression:

$$
v_{p}^{*} \quad \frac{(1 \rho) d_{p}}{T_{V}\left(b+d_{p}+\alpha k_{w} C^{*}\right)} \sigma+\frac{\beta W^{*}}{b+d_{p}+\alpha k_{w} C^{*}} .
$$

Equation (14) can be understood as follows: the first term cor responds to the $E$. coli load that remains on the poultry during the chiller process. That is, $\left(\begin{array}{ll}1 & \rho\end{array}\right) d_{p} /\left(T_{V}\left(b+d_{p}+\alpha k_{w} C^{*}\right)\right)$ quantifies the fraction of the incoming $E$. coli load on the poultry that does not shed during chilling and is not inactivated by FC contact with carcass surfaces. The second term quantifies the $E$. coli load gained via cross contamination from contaminated chiller water. Recall that $\beta$ is the water to carcass transmission parameter, $1 / b$ is the characteristic time scale for $E$. coli shedding from carcasses into the chiller water (it is proportional to the time it takes to shed $1-2 \log _{10}$ CFU) and $1 / d_{p}$ is the average dwell time of a carcass in the chiller tank. Notice that $1 /\left(\alpha k_{w} C^{*}\right)$ is the characteristic time scale for FC to inactivate E. coli on carcass surfaces when the FC has reached the equilibrium amount of $C^{*}$. Combining these three time scales, we observe that $1 /\left(b+d_{p}+\alpha k_{w} C^{*}\right)$ is the characteristic time scale of cross contamination during an $8 \mathrm{~h}$ shift of continuous processing. In other words, some of the E. coli gained from cross contamination may be shed or inactivated before the carcass leaves the chiller tank and the model accounts for this by shortening the effective cross contamination time scale from $1 / d_{p}$ to $1 /\left(b+d_{p}+\alpha k_{w} C^{*}\right)$.

While $v_{p}^{*}$ is sensitive to many parameters, referring to Fig. 1(C), we see that $c_{1}, \alpha, l$ and $q$ play more influential roles. In terms of chlorine efficacy, Fig. 1 indicates that $c_{1}$ (the input FC concentration) has a strong effect on reducing the $E$. coli load on carcasses during chilling. This effect is coupled with the impact of $l$ (the input of fresh water/carcass) as increasing $l$ increases the addition rate of FC to the tank (see formula (6)). From an industry standpoint, it is important to note that both $c_{1}$ and $l$ can be directly controlled during the chilling process. Additionally, from a regulatory perspective, $c_{1}$ has an upper bound. Considering this limitation, we will give a more detailed discussion of FC control as well as discuss the role of $\alpha$ in Section 3.2.3. In terms of water usage and the parameter $l$, an interesting study would be to use the model (7) predictions to compare the tradeoffs between simultaneously minimizing $E$. coli loads in the red water and on carcass surfaces and the cost asso ciated to water consumption.

Fig. 1(C) suggests that an increase in $q$ (the fraction of organic material the sheds from carcasses into the water) leads to an in crease in $v_{p}^{*}$. While $q$ cannot be directly controlled during chilling, as 
Table 3

Parameters and ranges for sensitivity analysis.

\begin{tabular}{|c|c|c|}
\hline Parameter/Reference & Baseline & Range \\
\hline $\begin{array}{l}\sigma \\
\text { (Cavani et al., 2010; Northcutt et al., 2008) }\end{array}$ & $2 \times 10^{4} \mathrm{CFU} / \mathrm{kg}$ & $10^{3} 10^{6}$ \\
\hline $\begin{array}{l}\rho \\
\text { Estimated }\end{array}$ & 0.15 & $0.05 \quad 0.30$ \\
\hline $\begin{array}{l}q \\
\text { (Northcutt et al., 2008; Tsai et al., 1992) }\end{array}$ & 0.011 & $0.005 \quad 0.03$ \\
\hline $\begin{array}{l}\beta \\
\text { (Munther \& Wu, 2013; Northcutt et al., 2008), estimated }\end{array}$ & $0.01(\mathrm{~kg} \mathrm{~min})^{1}$ & 0.0010 .1 \\
\hline $\begin{array}{l}b \\
\text { (Northcutt et al., 2008) }\end{array}$ & $0.077 \min { }^{1}$ & $0.04 \quad 0.1$ \\
\hline $\begin{array}{l}\alpha \\
\text { estimated }\end{array}$ & 0.05 & 0.0010 .1 \\
\hline $\begin{array}{l}k_{w} \\
\text { (Helbling \& VanBriesen, 2007; Zhang et al., 2015) }\end{array}$ & $216 \mathrm{l} /(\mathrm{mg} \min )$ & 150300 \\
\hline $\begin{array}{l}\text { l } \\
\text { (Northcutt et al., 2008; CFIA, 2014) }\end{array}$ & $1.7 \mathrm{l} /$ carcass & 14 \\
\hline $\begin{array}{l}c_{1} \\
\text { (USDA, 2012; CFIA, 2014) }\end{array}$ & $25 \mathrm{mg} / \mathrm{l}$ & 050 \\
\hline $\begin{array}{l}h_{J} \\
\text { (Tsai et al., 1992) }\end{array}$ & $0.0017(\mathrm{~kg} \mathrm{~min})^{1}$ & 0.00110 .0022 \\
\hline
\end{tabular}

(A)

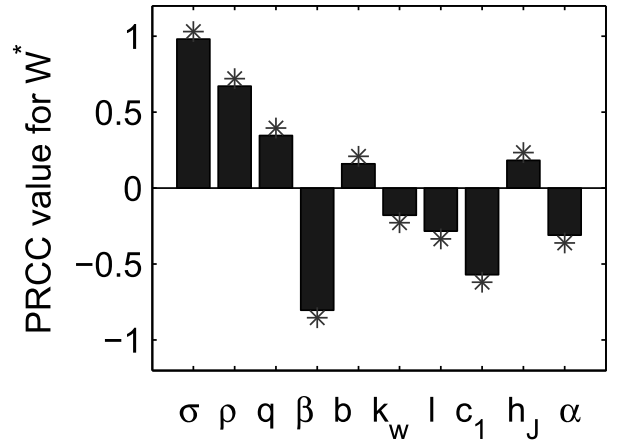

(C)

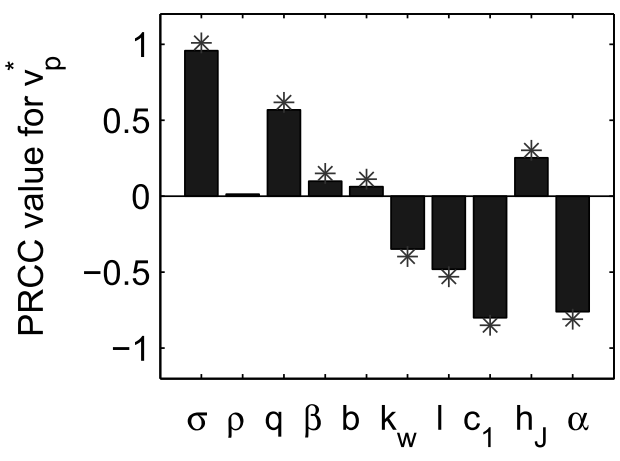

(B)

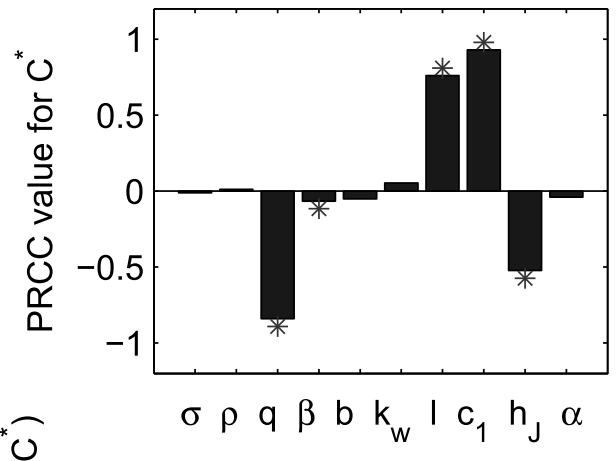

(D)

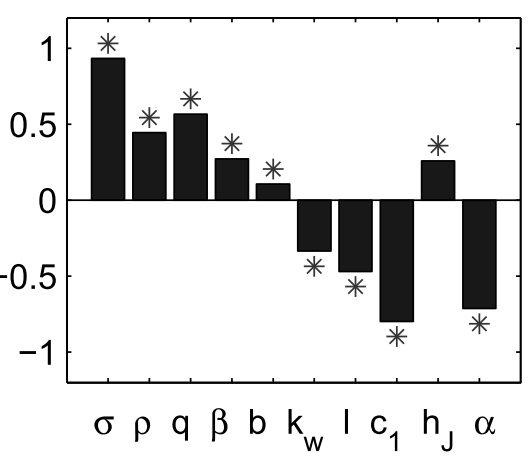

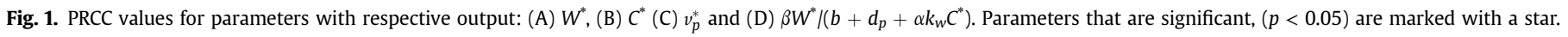

in the case of $c_{1}$ and $l$, it can be indirectly regulated during pre chiller processing. See Section 3.2.3 for more details on the rela tionship between $q$, FC control and pre chiller processing interventions.

\subsubsection{Rules of thumb for E. coli cross contamination}

Under what conditions should we worry about cross contamination? In terms of Equation (14), this question translates into comparing the magnitude of the first term and the cross contamination term. Since $c_{1}$ (the input FC concentration) can be controlled during processing, we estimate the magnitude of the fraction $(1 \quad \rho) d_{p} /\left(T_{V}\left(b+d_{p}+\alpha k_{w} C^{*}\right)\right)$, the cross contamination term, $\beta W^{*} /\left(b+d_{p}+\alpha k_{w} C^{*}\right)$, and the $E$. coli level in water $W^{*}$ as follows: for each fixed $c_{1}$ in $[0,50] \mathrm{mg} / \mathrm{l}$, we perform Monte Carlo simulations to calculate the respective values of these terms. Then, fitting a normal distribution to the range of respective outputs, we calculate the $95 \%$ confidence interval.

The results are illustrated in Fig. 2. Examining Fig. 2(A), we see that the 95\% confidence interval for $(1 \rho) d_{p} /\left(T_{V}\left(b+d_{p}+\alpha k_{w} C^{*}\right)\right)$ ranges over approximately $\left[10^{-10.4}, 10^{-8.4}\right]$ as $c_{1}$ varies from $[0,50]$. 
Similarly, from Fig. 2(B) and (C), the cross contamination term ranges over $\left[10^{-6.75}, 10^{-3.3}\right]$ and $W^{*}$ ranges over $\left[10^{-4.4}, 10^{-3}\right]$, respectively. These results provide quantifiable evidence that FC plays a significant role in reducing the $E$. coli load on poultry both by directly inactivating the bacteria on carcass surfaces and by inac tivating the bacteria in the chiller water which reduces the load gained via cross contamination.

However, the question remains, when should we worry about cross contamination? Using the results from Fig. 2(A) and (B), we can compare the expressions for $v_{p}^{*}$ when $c_{1} \quad 0$ (no FC input) and when $c_{1} 50$ (maximum FC input). First, for $c_{1} \quad 0$, we have that

$v_{p}^{*} \approx 10^{-8.4} \sigma+10^{-3.3}$

Next, for $c_{1} 50$, we see that

$v_{p}^{*} \approx 10^{-10.4} \sigma+10^{-6.75}$

From Equation (15), if $\sigma \leq 10^{5.1}$, the magnitude of the cross contamination term plays a dominant role in determining the overall order of $v_{p}^{*}$. That is, during a typical $8 \mathrm{~h}$ shift, if no FC is used, cross contamination has a primary effect in determining the $E$. coli level on post chiller poultry when the average $E$. coli load on pre chiller carcasses is on the order of $5 \log _{10}$ CFU or less.

Following the same reasoning, Equation (16) indicates that if $\sigma \leq 10^{3.7}$, then the cross contamination dynamic is again signifi cant. In other words, during a typical $8 \mathrm{~h}$ shift, if maximum FC input is used, cross contamination plays a leading role in determining the $E$. coli level on post chiller poultry when the average $E$. coli load on pre chiller carcasses is on the order of $4 \log _{10}$ CFU or less. Therefore, while FC input significantly reduces $E$. coli levels in the water and on poultry during chilling, from a management perspective, it plays a lesser role in ensuring that cross contamination will not be an issue. That is, using maximum FC input, compared to zero FC input, reduces the range of $\sigma$, within which cross contamination dictates the magnitude of $v_{p}^{*}$, only by about $1 \log _{10} \mathrm{CFU}$.

\subsubsection{Cross contamination and flock to flock transmission}

Our analysis also indicates that $W^{*}$ is sensitive to $\beta, \rho$, and $b$, and $\beta W^{*} /\left(b+d_{p}+\alpha k_{w} C^{*}\right)$ is sensitive to $\rho$ and $b$ (see Fig. $1(\mathrm{~A})$ and (D)). This information coupled with our discussion in Section 3.2.1 points to potential cross contamination issues as described in the following archetypal situation: Suppose chickens are processed from a variety of farms at one particular processing center but farm (A), at some juncture, delivers chickens that carry a significantly higher E. coli load as compared with the chickens from the other farms. It is likely then, that the $E$. coli load in the chiller water, $W^{*}$, will dramatically increase via the shed from farm (A) chickens during chilling. If the magnitude of $W^{*}$ is higher or comparable to the magnitude of $\sigma$ from carcasses now entering the chiller tank, and the chiller water has yet to be replaced, then cross contamination (flock to flock) may be significant. To obtain rules of thumb for such scenarios, that are backed by scientific rigor at the mechanistic scale, our model suggests the need for specific experiments to capture the components of shedding ( $\rho$ and $b$ ) and cross contamination $(\beta)$ more precisely. Refer to Section 4 for a more detailed discussion.

\subsubsection{FC control and inactiviation}

While our findings in Section 3.2.1 show that cross contamination can strongly influence the resulting $E$. coli load on chilled poultry, both in the presence or absence of FC input, the results in Figs. 1 and 2 demonstrate that FC input is pivotal as a control. To quantify this control on E. coli levels, we again consider
(A)

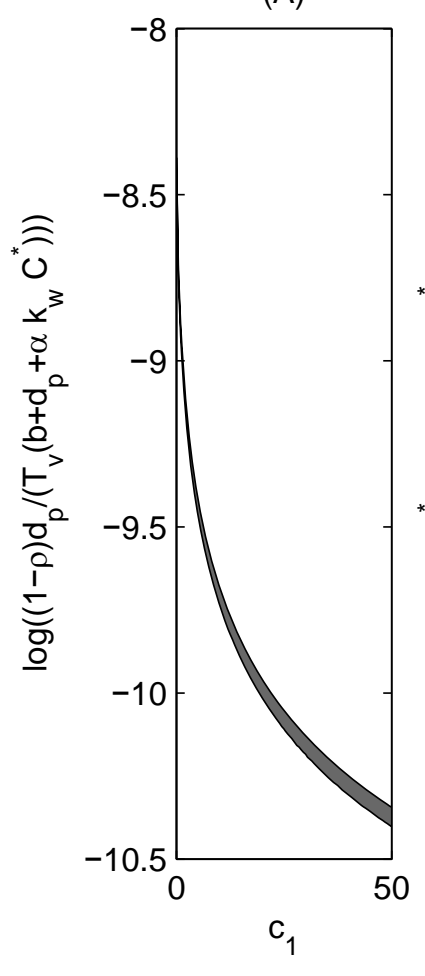

(B)

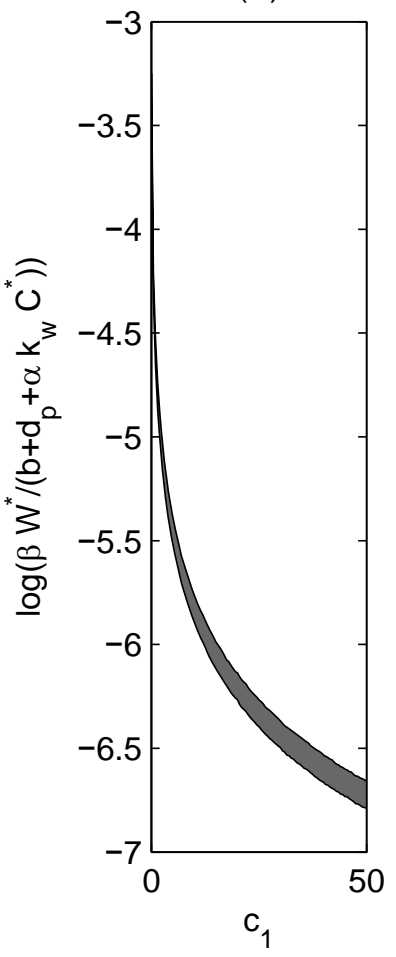

(C)

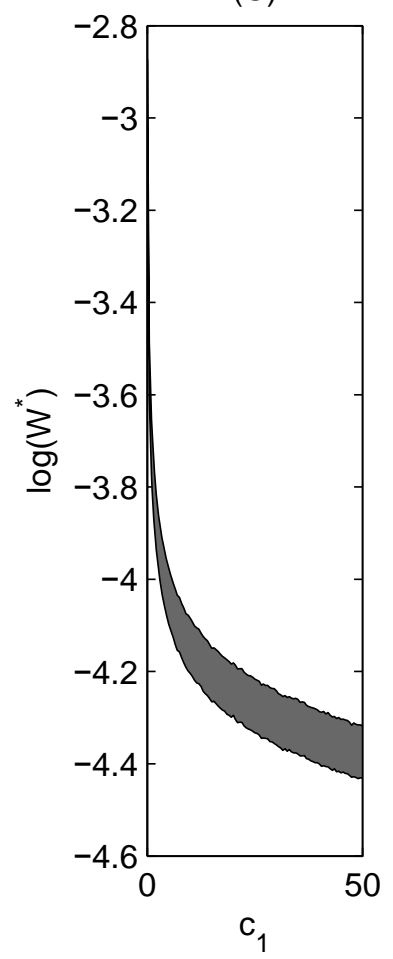

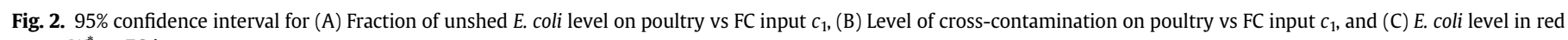
water $W^{*}$ vs FC input $c_{1}$. 
Equation (14). Rescaling $v_{p}^{*}$ to have units CFU/carcass and rescaling $\sigma$ to have units CFU/carcass, we let $v_{p}^{*} 2 T_{V} v_{p}^{*}$ (CFU/carcass) and $\sigma^{*} \quad 2 \sigma$ (CFU/carcass) where $T_{V}$ is the tank volume and each carcass is assumed to be $2 \mathrm{~kg}$ on average. Therefore, (14) becomes:

$$
\tilde{v}_{p}^{*} \quad 2 T_{V} v_{p}^{*} \quad \frac{(1 \rho) d_{p}}{\left(b+d_{p}+\alpha k_{w} C^{*}\right)} \sigma^{*}+\frac{2 T_{V} \beta W^{*}}{b+d_{p}+\alpha k_{w} C^{*}}
$$

Recall in Section 2.3, we assume that FC is mixed with fresh water input at the entrance to the chiller tank. Connecting to our model, we want to understand how the FC input, captured by the parameter $c_{1}$, affects the microbial level on outgoing poultry, given by $v_{p}^{*}$ in (17). Note that because solutions equilibrate well before a typical $8 \mathrm{~h}$ shift ends, we focus on the time independent value $v_{p}^{*}$.

The USDA and CFIA both specify that at most $50 \mathrm{mg} / \mathrm{l}$ of FC input can be used during chilling. Also, the USDA (USDA, 2012) examined E. coli test levels from national baseline studies concerning poultry slaughter establishments and has categorized these levels into three control ranges: Acceptable $(<100 \mathrm{CFU} / \mathrm{ml})$, Marginal $(100-1000 \mathrm{CFU} / \mathrm{ml})$ and Unacceptable $(>1000 \mathrm{CFU} / \mathrm{ml})$, where a $400 \mathrm{ml}$ solution is used in the rinse procedure to remove microbes from the carcass surface. For the units CFU/carcass, these ranges translate to.

- Acceptable ( $<4 \times 10^{4} \mathrm{CFU} /$ caracass)

- Marginal ( $4 \times 10^{4}$ to $4 \times 10^{5} \mathrm{CFU} /$ carcass)

- Unacceptable (>4 × $10^{5} \mathrm{CFU} /$ carcass).

To illustrate our model results in the context of these ranges, we run simulations for two scenarios: (1) when $c_{1} \quad 0$ (i.e. no FC input) and (2) when $c_{1} \quad 50 \mathrm{mg} / \mathrm{l}$. Specifically, for each $\sigma^{*}$ in $\left[2 \times 10^{3}, 2 \times 10^{6}\right] \mathrm{CFU} /$ carcass, we perform Monte Carlo simulations for $v_{p}^{*}$, fit a normal distribution to the outputs and calculate the $95 \%$ confidence interval. Fig. 3 displays the model outcomes. Notice that the confidence intervals for all scenarios are quite narrow (this is partly because of the log scale on both axes). The region below the green line $A$ illustrates Acceptable $E$. coli levels, the region between the green line $A$ and the red line $M$ illustrates Marginal levels and the region above the red line represents Unacceptable levels. The $C_{0}$ "line" represents $v_{p}^{*}$ for no FC input and the $C_{50}$ "lines" represent $v_{p}^{*}$ under $50 \mathrm{mg} / \mathrm{l}$ of $\mathrm{FC}$ input, corresponding to the respective values for $\alpha$.

Fig. 3 illustrates the sensitivity of $v_{p}^{*}$ to $\alpha$, corresponding to Fig. 1(C). Recall that $\alpha$ comes from the term $\alpha k_{w}$ which captures the rate of $E$. coli inactivation via FC contact with carcass surfaces. Because we have no data to determine this rate, we estimated $\alpha \in$ [0.001,0.1] (see the discussion in Section 2.2 for more details). While the uncertainty associated with $\alpha$ leads to uncertainty in the direct quantification of $v_{p}^{*}$, the results in Fig. 3 ensure that no matter the value of $\alpha$, with maximum FC input, the $E$. coli load on poultry during chilling may be reduced by about $1-3 \log _{10}$ CFU/carcass. To narrow this range, experiments are needed to estimate the rate $\alpha k_{w}$.

However, in terms of USDA control ranges, Fig. 3 confirms that whether we know the exact value of $\alpha$ or not, maximum FC input will keep the $E$. coli level on post chiller carcasses below the Un acceptable range and most likely within the Acceptable range when $\sigma^{*}<2 \times 10^{6} \mathrm{CFU} /$ carcass. This can be seen in Fig. 3 where the $C_{50}$ line remains below the red line $M$ for all values of $\alpha$, and remains below the green line $A$ for $\alpha \quad 0.1$ and 0.01 .

In line with FC control, it is worth noting that $C^{*}$, the FC level in the chiller water, is sensitive to a number of parameters (see Fig. 1(B)). The freshwater input rate $l$, and $c_{1}$ clearly have a signifi cant positive impact on $C^{*}$. What is noteworthy is that $q$ and $h_{J}$ both significantly decrease the FC level. Recall that

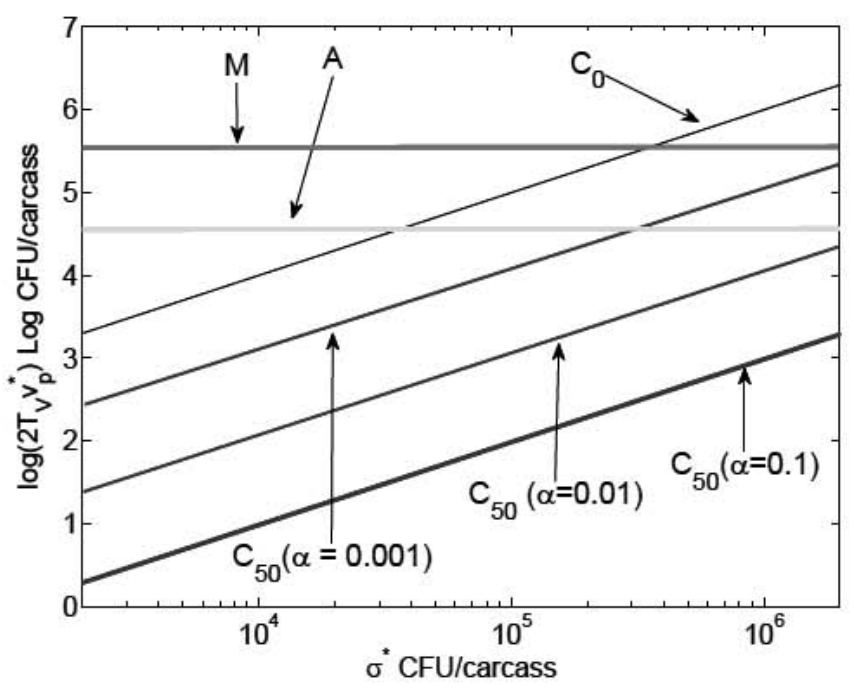

Fig. 3. FC control of $E$. coli on poultry during chilling in terms of pre-chill contamination levels: On the vertical axis, $\log \left(2 T_{v} v_{p}^{*}\right)$ corresponds to $\log _{10}\left(v_{p}^{*}\right) . C_{0}$ illustrates the (very narrow) $95 \%$ confidence interval for average $E$. coli load on post-chiller carcasses with $c_{1} \quad 0 \mathrm{mg} / \mathrm{F} \mathrm{FC}$ input and $C_{50}$ illustrates $95 \%$ confidence interval for average E coli load on post-chiller carcasses with $c_{1} 50 \mathrm{mg} / \mathrm{IFC}$ input for $\alpha \quad 0.1,0.01$, and 0.001 , respectively. Line $A$ (green) is the "Acceptable" $E$. coli load/carcass and line $M$ (red) is the "Marginal" E. coli load/carcass as specified by the USDA. (For interpretation of the references to colour in this figure legend, the reader is referred to the web version of this article.)

$$
C^{*} \frac{c_{1} g}{\left(10^{-3} T_{V}\right)\left(d_{c}+k_{c} W^{*}+\alpha k_{c} v_{j}^{*} J^{*}+\alpha k_{c} v_{p}^{*} P^{*}+h_{J} J^{*}+\frac{g}{10^{3} T_{V}}\right)} .
$$

Since the relative magnitude of $d_{c}$ is small (i.e. FC degrades relatively slowly), the magnitude of all the terms involving $k_{c}$ is small as a limited amount of FC is needed to neutralize $E$. coli in solution and on carcass surfaces, and the order of $g /\left(10^{-3} T_{V}\right)$ is small (flow out), the effective magnitude of $C^{*}$ is given by:

$C^{*} \approx \frac{C_{1}}{h_{\jmath} J^{*}}$

where we let $C_{1} \quad c_{1} g /\left(10^{-3} T_{V}\right)$. This means that the organic material (blood, fat, protein, ingesta, etc.) in the chiller water plays an important role in neutralizing the effect of FC. This notion is supported in the literature (Russell, 2012). Furthermore, as $J^{*}$ depends on $q$, Equation (18) illustrates how $C^{*}$ is sensitive to $q$. Essentially $q$ is a measure of how "clean" the carcasses are as they enter the chiller tank. For instance, if over scalding occurs $q$ may be much higher due to the excessive fat that will shed from the carcasses during chilling (Russell, 2012). In this case, the FC level decreases and our model indicates that cross contamination may be an issue for a wider range of $E$. coli loads on incoming carcasses (i.e. for a larger range of $\sigma$ ). Note that results from Tsai et al. (1992) describe chlorine consumption in poultry chiller water as a function of the amount of lipids and other components shed from carcasses into the water. Our model predictions are supported by the combination of results from Tsai et al. (1992) and results from Mead and Thomas (1973), which reported that efficacy of chlorine to control microbial levels during poultry chilling depends, in particular, on the chemical composition of the process water. 


\section{Discussion and future directions}

Although cross contamination during immersion chilling in volves complex phenomena, our model (7) is able to simplify these dynamics for relatively easy assessment. In the case of generic E. coli contamination, solutions to our model reach an equilibrium state on the order of 200250 minutes. This means that during a typical $8 \mathrm{~h}$ shift, if the average $E$. coli load on poultry entering the chill tank $(\sigma)$ is relatively constant, we can use the equilibrium solutions (see Equations $8-12$ ) to predict $E$. coli levels for instance, on poultry exiting the chiller, $v_{p}^{*}$. The advantage here is that $v_{p}^{*}$ is given by an analytic expression in terms of model parameters. Thus, our model provides a pragmatic, quantified description of $E$. coli cross contamination in terms of processing and control parameters.

As mentioned in Section 3.2.1, we find that if $\sigma$ has a magnitude of least $4 \log _{10} \mathrm{CFU}$, cross contamination may not affect the con centration of $E$. coli on post chill carcasses as significantly as when the incoming concentration is less than $4 \log _{10}$ CFU. This suggests that maximum FC input may be unsuccessful in preventing cross contamination, placing the emphasis on surveillance of pre chiller contamination. On the other hand, Figs. 1-3 reveal that FC input is still able to significantly reduce the $E$. coli load on poultry during chilling.

However, if the model (7) is to capture, for instance, significant flock to flock cross contamination, $\sigma$ should be a function of time, determined by appropriate data. In particular, by varying $\sigma$ in time, we can extend the model (7), which we plan to explore in a future paper, to be used as a reference point to inform strategies for flock processing throughout a given day. Results from such a model can inform logistical slaughter, a processing strategy that orders flocks with greater incoming concentrations of $E$. coli or other bacteria to be processed last. In contrast to the model (7), solutions of the extended model may not settle to equilibrium. While this situation is more complicated, the model will still mechanistically link pro cessing and control parameters to bacterial contamination in the chiller water and on chiller carcasses. This implies that model pa rameters can be tuned in order to keep contamination levels within certain bounds, and this interplay will offer insight towards control strategies. In line with this, we stress the importance of the results from the model (7). It is critical to note that even if $\sigma$ varies in time, if $\sigma(t) \geq 4 \log _{10}$ CFU or $\sigma(t)<4 \log _{10}$ CFU throughout the course of an $8 \mathrm{~h}$ shift, our rules of thumb for generic E. coli cross contamination (e.g. in the case of maximum FC input in Section 3.2.1) still hold. Thus, this illustrates how the model (7) can provide a key man agement threshold for addressing cross contamination issues.

In addition to providing insight toward cross contamination management, our model (7) and the extended model are useful for QMRA during poultry processing. Ideally, control strategies should be built on knowledge of both prevalence and concentration of contamination during processing. While stochastic models are the typical players used to address these concerns, microbial transfer coefficients at various steps may be unknown or loosely estimated, limiting the confidence in predictive results. The advantage of our model (7) and the extended model, is that both can be used in QMRA to set baseline parameter values and func tional forms for bacterial transfer coefficients that are data informed, rooted in mechanistic foundations and may be hard to precisely measure via experiments. Thus our modeling approach can bolster the confidence in the risk predictions from such ana lyses. Furthermore, these models, as opposed to expensive exper iments, can provide quantitative evidence as to which assumptions should or should not be included in large scale risk models during poultry processing. For example, should a risk model of poultry processing include, at the chill step, the effects of the organic ma terial $(J)$ in both neutralizing FC and in determining bacteria levels on carcasses and in the process water in during chilling? Rather than conducting multiple experiments to estimate the probabilities of how these mechanisms affect contamination levels in the tank, our model (7) outputs based on selected inputs can be used to estimate microbial transfer with or without the consideration of the organic load (i.e. with $h_{J}>0$ or $h_{J} \quad 0$, respectively).

Also, our approach allows for testing the sensitivity of our model outputs relative to specific model parameters, and therefore, pro vides guidance for specific future experimentation. In terms of bacterial transfer, our model (7) results indicate, see Fig. 1(A) and (D), that the E. coli level in the red water and the E. coli load gained by poultry via cross contamination are sensitive to $\rho$ and $b$ (carcass to water shed rates) and $\beta$ (water to carcass transmission rate) and therefore, in the case where $\sigma$ may vary over multiple orders of magnitude during an $8 \mathrm{~h}$ shift, the parameters $\rho, b$ and $\beta$, need to examined in more detail before being applied to the extended model. For instance, data for the probability of microbial attachment $\mu$, on which $\beta$ depends, as well as details connecting water flow through the tank, sheer forces in the red water to $\rho$ and $b$, would be necessary to have greater accuracy and predictability in understanding the dynamics of the chiller tank. Furthermore, if the carcass to water ratio is sufficiently high, the model should include carcass to carcass type transmission. Again, in order to quantify such transmission during chilling, further experiments are needed.

Another mechanism that may contribute to the E. coli load on carcasses during chilling is carcass surface temperature. Results from Carciofi and Laurindo (2007) indicate that the average tem perature just under the skin of a $2 \mathrm{~kg}$ carcass, subject to water temperature similar to our modeling assumptions, takes between 5 and $10 \mathrm{~min}$ on average to cool from its prechill temperature (be tween 33 and $40^{\circ} \mathrm{C}$ ) to $4 \mathrm{C}$. While bacterial growth could pre sumably occur during this time, data from the study in Northcutt et al. (2008), subject to similar chill tank operating conditions as in our model (7), indicate that this growth most likely is not sig nificant. For instance, for $E$. coli, they found that the average prechill level on carcasses was $2.6 \log _{10} \mathrm{CFU} / \mathrm{ml}$, the average postchill level was at most $1.2 \log _{10} \mathrm{CFU} / \mathrm{ml}$, and the level in the chiller water was at most $1.2 \log _{10} \mathrm{CFU} / \mathrm{ml}$ (Northcutt et al., 2008). This suggests that there is no significant growth of bacteria on the carcass surface during the cooling phase, and therefore, we do not include this mechanism in the model (7).

While we have discussed contamination mechanisms that need more exploration and mechanisms that may be ignored, an important feature of our model is that it can quantify the effect of indirect mechanisms involved with cross contamination. For example, it is known that organic material such as blood, fat, pro tein, and digesta react with FC in the red water, reducing its efficacy to eliminate microbes (Russell, 2012). However, from the model (7) we can obtain the FC concentration in the red water explicitly in terms of model parameters. In particular, we have developed an expression (Equation (18)) which links FC reduction to the E. coli and organic material load of the poultry entering the tank and the rate at which organic material reacts with FC. This reinforces the importance of reducing the organic material on poultry during the pre chilling stages of processing. For instance, spraying procedures along the evisceration line, pre scald mechanisms and the scalding process, are typical practices that affect the organic load in the chill tank (Russell, 2012).

Generally speaking, we have shown how microbiological data for generic E. coli can inform the model (7) in order to understand cross contamination mechanisms as well as quantified control limits. In terms of future directions, the model (7) can be used for similar analyses involving human pathogens such as Campylobacter, Salmonella, and E. coli 0157:H7, using relevant data for pathogen specific parameters. In addition, our model framework can be 
adapted to describe industrial scale immersion chilling operations in the U.S. and other locations by modifying certain processing parameters. Referring to Table 2, this may involve adjusting type (i) parameters as well as type (ii). Even if the immersion chilling process involves slightly different practices, such as recycling of red water, our modeling approach can be adjusted to account for these mechanisms.

Finally, it would be desirable to stratify poultry into different categories based on pathogen loads (perhaps in terms of thresholds highlighted by the USDA baseline studies). From this perspective, stochastic/agent based simulations (discrete models) can be used to more precisely examine flock to flock cross contamination and derive rules of thumb for logistical slaughter in terms of both prevalence and level of contamination. The model (7) is a vital tool for parameterizing these new discrete models in the context of poultry contamination with human pathogens.

\section{Acknowledgments}

This work was partially supported by the Natural Sciences and Engineering Research Council of Canada (Grant number: 105588 2011), the Canada Research Chairs Program (Grant number: 230720), Mitacs and the Mprime Centre for Disease Modelling. The authors sincerely thank the two anonymous reviewers for their careful reading and helpful suggestions for improving the exposi tion of the article. Finally, the authors want to thank the CFIA for their support and details concerning poultry slaughter establish ments which operate under the CFIA approved Modernized Poultry Inspection Program.

\section{References}

Adams, M. R., Hartley, A. D., \& Cox, L. J. (1989). Factors affecting the efficacy of washing procedures used in the production of prepared salads. Food Microbiology, 6, 6977.

Brauer, F. (2008). Compartmental models in epidemiology. In F. Brauer, P. van den Driessche, \& J. Wu (Eds.), Mathematical epidemiology. Berlin: Springer-Verlag.

Bucher, O., Farrar, A. M., Totton, S. C., Wilkins, W., Waddell, L. A., WIhelm, B. J., et al. (2012). A systematic review-meta-analysis of chilling interventions and a metaregression of various processing interventions for salmonella contamination of chicken. Preventive Veterinary Medicine, 103, 115.
Carciofi, B. A. M., \& Laurindo, J. B. (2007). Water uptake by poultry carcasses during cooling by water immersion. Chemical Engineering and Processing, 46, 444450.

Carrasco, E Morales-Rueda, A. \& Garcia-Gimeno, R. M. (2012). Cross-contamination and recontamination by salmonella in foods: a review. Food Research International, 45, 545556.

Cavani, R., Schocken-Iturrino, R. P., Garcia, T. C. F. L., \& de Oliveira, A. C. (2010). Comparison of microbial load in immersion chilling water and poultry carcasses after 8,16 and 24 working hours. Ciencia Rural, 40, 16031609.

CFIA. (2014). Poultry inspection programs. http://www.inspection.gc.ca/food/meatand-poultry-products/manual-of-procedures/chapter-19/eng/1360962146879/ 1360962607138 Accessed: March 2015.

Deborde, M., \& von Gunten, U. (2008). Reactions of chlorine with inorganic and organic compounds during water treatment kinetics and mechanisms: a critical review. Water Research, 42, 1351.

Gil, M. I., Selma, M. V., López-Gálvez, F., \& Allende, A. (2009). Fresh-cut product sanitation and wash water disinfection: problems and solutions. International Journal of Food Microbiology, 143, 3745.

Helbling, D. E., \& VanBriesen, J. M. (2007). Free chlorine demand and cell survival of microbial suspensions. Water Research, 41, 44244434.

Li, X., Gu, D., Qi, J., Ukita, M., \& Zhao, H. (2003). Modeling of residual chlorine in water distribution system. Journal of Environmental Sciences, 15, 136144.

Marino, S., Hogue, I. B., Ray, C. J., Krischner, D. E., \& Zhao, H. (2008). A methodology for performing global uncertainty and sensitivity analysis in systems biology Journal of Theoretical Biology, 254, 178196.

Mead, G. C., \& Thomas, N. L. (1973). Factors affecting the use of chlorine in the spin chilling of eviscerated poultry. British Poultry Science, 14, 99117.

Munther, D., \& Wu, J. (2013). Enhanced surveillance on food-borne disease outbreaks: dynamics of cross-contamination in biocidal wash procedure. Journal of Theoretical Biology, 321, 2835.

Northcutt, J. K., Smith, D., Huezo, R. I., \& Ingram, K. D. (2008). Microbiology of broiler carcasses and chemistry of chiller water as affected by water reuse. Poultry Science, 87, 14581463.

Ratkowsky, D. A., Olley, J., McMeekin, T. A., \& Ball, A. (1982). Relationship between temperature and growth rate of bacterial cultures. Journal of Bacteriology, 149, 15.

Russell, S. M. (2012). Controlling Salmonella in poultry production and processing. Boca Raton, FL: CRC Press.

Thomas, C. J., \& McMeekin, T. A. (1980). Contamination of broiler carcass skin during commercial processing procedures: an electron microscopic study. Applied and Environmental Microbiology, 40, 133144.

Tsai, L. S., Schade, J. E., \& Molyneux, B. T. (1992). Chlorination of poultry chiller water: chlorine demand and disinfection efficiency. Poultry Science, 71 188196.

USDA. (2012). Fsis directive, verifying sanitary dressing and process control procedures by off-line ipp in poultry slaughterhouse operations, effective October 28, 2012 http://www.fsis.usda.gov Accessed: January 2014.

Wang, G., \& Doyle, M. P. (1998). Survival of enterohemorrhagic Escherichia coli o157: h7 in water. Journal of Food Protection, 6, 657775.

Zhang, B., Luo, Y., Zhou, B., Wang, Q., \& Millner, P. D. (2015). A novel microfluidic mixer-based approach for determining inactivation kinetics of Escherichia coli o157:h7 in chlorine solutions. Food Microbiology, 49, 152160. 